Revista de Psicologia de la PUCP. Vol. XV11, 2, 1999.

\title{
ELECCIÓN VOCACIONAL Y PERSONALIDAD EN UNIVERSITARIOS A TRAVÉS DEL PSICODIAGNÓSTICO DE RORSCHACH ${ }^{1}$
}

\author{
Patricia Martínez U. ${ }^{2}$ \\ Pontificia Universidad Católica del Perí
}

El presente estudio se centró en el análisis de la relación entre personalidad y elección vocacional en jóvenes universitarios del último año de estudios de una universidad particular de Lima. El objetivo fue describir y comparar las características de personalidad más saltantes en cuatro facultades distintas: ingeniería, economía, derecho y educación, de donde se obruvo un total de 120 participantes. Para ello se utilizó el Psicodiagnóstico de Rorschach, codificado e interpretado de acuerdo al Sistema Comprehensivo de Exner. Los resultados indicaron tendencias o estilos de respuesta distintos en los cuatro grupos, además de características comunes en todos ellos. Destacó el grupo de ingeniería, con un estilo cognitivo particularmente diferente al de los otros grupos.

Vocational choice and personality in university students with the Rorschach test This study, carried out in 120 last year students of a private university in Lima, focuses in the relationships between personality and vocational choice. The objective of rhe study was to describe and compare the most significant personality characteristics of students from four different academic programs: Engineering, Economics, Law and Education. The Rorschach Psychodiagnostic, coded and interpreted according to the Exner Comprehensive System was used for this purpose. Resulss showed that, in spite of the common characteristics, the response tendencies and styles were different in the four groups, particularly in the Engineering, that showed a cognitive style significantly different from the other groups.

1. Una versión de esta investigación se presentó en el Il Congreso Iberoamericano de Evaluación Psicológica en Junio de 1999, en Caracas, Venezucla.

2. Magister en Psicología de la Pontificia Universidad Católica del Perú, donde se desempeña además como Profesora Asociada del área Clínica de la especialidad de Psicología y como Coordinadora ante la Facultad de Letras y Ciencias Humanas. Correo electrónico: pmartin@pucp.edu.pe. 

Una de las decisiones más importantes en la vida es la elección de una determinada carrera u ocupación, es por ello que ha sido a lo largo de los años un tema de gran interés para muchos investigadores en el campo de la Psicología.

El que elige no está eligiendo sólo una carrera, está eligiendo con qué trabajar, está definiendo para qué hacerlo, está pensando en un sentido para su vida, está eligiendo un cómo, delimitando un cuándo y un dónde. Está definiendo quién ha de ser y elaborando su proyecto de vida, por lo tanto está estrechamente ligada al tema de la Identidad.

El logro de la identidad (Erikson, 1993), supone una integración yoica de comportamientos y sentimientos diversos con relación a los distintos roles que se tienen que desempeñar. Bohoslavsky (1984), se refiere a la identidad ocupacional como un aspecto ligado al desarrollo de la identidad que sólo puede separarse arbitrariamente de la identidad sexual o familiar, o de cualquier otro aspecto de la misma, ya que la personalidad o la identidad, funcionan como un todo integrado. Sin embargo, el criterio de totalidad no implica el de homogeneidad de las partes y es, precisamente, la heterogeneidad la que permite diferenciar problemas familiares, de problemas de pareja o de problemas vocacionales. Se trata de niveles de análisis distintos que, como marcos referenciales, permiten diferenciar todo aquello relacionado con la identidad vocacional $\mathrm{u}$ ocupacional.

En este contexto, todo conflicto ante la elección de una manera de ser mediante una ocupación expresa una dificultad en la integración de identidades diversas. Así, todas las dudas obedecen a identificaciones no integradas. La identidad ocupacional será considerada entonces, como un momento en un proceso que se encuentra sometido a las 
mismas leyes y dificultades que aquél que conduce al logro de la identidad personal.

Casullo et al. (1996), se refieren a la construcción de un proyecto de vida que se encuentra estrechamente ligado a la identidad ocupacional, entendida como la representación subjetiva de la inserción en el mundo del trabajo. Así, un proyecto de vida requiere de la elaboración y consolidación de una identidad ocupacional, que, generalmente se plantea en términos del yo quisiera ser, o yo quisiera hacer. La construcción o elaboración de un proyecto de vida forma parte del proceso de maduración afectiva e intelectual y como tal supone un aprender a crecer.

La identidad ocupacional, así como la identidad personal tienen que entenderse como la continua interacción entre factores internos y externos a la persona. En toda profesión hay una relación con objetos que están fuera del espacio propio, que pertenecen a un espacio ajeno a nosotros pero con los cuales nos relacionamos a partir de nosotros (Holland, 1996). El autor hace referencia a la identidad vocacional como un concepto que puede explicar, mejor que el de los intereses, la estabilidad o inestabilidad de una carrera, y la satisfacción que se encuentre en ella.

El sentimiento de identidad ocupacional se desarrolla sobre la base de las relaciones con los otros. Según Bohoslavsky (1984), para comprender la elección vocacional de una persona se requiere, entre otras cosas, analizar sus vínculos con el otro, porque la elección siempre tiene que ver con los otros. El futuro nunca es pensado en abstracto, nunca se piensa en una carrera o actividad despersonificadas. Siempre será esa carrera, o esa facultad o ese trabajo que cristaliza relaciones interpersonales pasadas, presentes y futuras.

La elección de una carrera es una síntesis compleja que hace intervenir numerosos elementos. Un oficio no es solamente una actividad, es un rango social, un nivel en una jerarquía, un medio de poner en práctica las aptitudes y de confirmar el valor social de las cualidades individuales (Super, 1977). 
Elección vocacional y personalidad en universitarios a través del psicodiagnóstico...

Existen múltiples propuestas teóricas para comprender el proceso de elección de una determinada carrera u ocupación, por lo tanto diversos tipos de clasificación. Todas las propuestas teóricas, están relacionadas de algún modo con las teorías de la personalidad, aunque con diferentes patrones sobre la relación entre personalidad y carrera (Osipow, 1991). Entre las más importantes se encuentran Roe (1972), Holland (1973, 1996), Super (1977) y Bohoslavsky (1984).

Sobre la base de estas teorías, se han realizado diversos estudios que intentan establecer conexiones entre personalidad y carrera, la mayor parte de ellos se refiere a la existencia de ciertos rasgos de personalidad que conducen a elegir una ocupación o a una gama de necesidades que buscan cubrirse a través de la carrera. En este sentido los patrones de interés representan diferentes modos de satisfacer necesidades personales. Se asume que las necesidades psicológicas se relacionan con los deseos de estudiar cierta carrera en la que se esperan satisfacer necesidades particulares.

La mayor parte de estudios tratan de establecer dicha conexión a través de distintos instrumentos, generalmente inventarios y pruebas de personalidad que evalúan rasgos específicos y que arrojan resultados variables. Entre los estudios más saltantes, se encuentran los realizados con la prueba de Holland y con la prueba de los Cinco Factores, entre ellos, se encuentran los estudios de Khan y Alvi (1991), Gottfredson, Jones y Holland (1993), Tokar y Swanson (1995) Furnham y Stringfield (1993), Nordvik (1996). La mayor parte de estos estudios encuentra una conexión entre ciertas características de personalidad y ciertos intereses u ocupaciones.

En el Perú, Ráez, Rossel, Niño de Guzmán, Martínez y Figueroa (1986), utilizaron el Psicodiagnóstico de Rorschach en su forma colectiva para la evaluación de grupos ocupacionales distintos. En sus resultados destacan la utilidad del instrumento para la evaluación de grupos y para la comparación de los mismos. Asimismo, afirman que la variable grado de instrucción es la que parece determinar mayores diferencias entre grupos. 
A partir de esto, nos preguntamos si existen características de personalidad, que puedan considerarse predominantes en ciertas carreras. Nuestro objetivo central es así, describir en detalle las características de personalidad más saltantes de los y las alumnas de cuatro facultades, y luego compararlos entre sí, con el fin de delimitar los aspectos en que se asemejan o diferencian.

\section{Metodología}

De acuerdo con los objetivos planteados, la presente investigación es de tipo Descriptivo-Comparativo, se trata de un diseño no experimental de tipo transeccional (Hernández, Fernández y Baptista, 1991), ya que se trata de describir cuatro grupos de alumnos evaluados en un mismo momento con el fin de compararlos.

\section{Participantes}

Se evaluaron 120 estudiantes de ambos sexos del último año de estudios universitarios, con una edad promedio de 24 años, pertenecientes a cuatro facultades de una universidad particular de Lima. Las facultades elegidas fueron: ingeniería electrónica, economía, derecho y educación. El criterio utilizado para la selección de ellas fue la cantidad de alumnos, ya que se trataba de las facultades más numerosas en esta universidad. De cada una de ellas se seleccionaron 30 alumnos mediante un muestreo no probabilístico de tipo incidental.

\section{Instrumento}

Se usó el Psicodiagnóstico de Rorschach, uno de los instrumentos más usados en la clínica y en la investigación, y que mayor información sobre el individuo proporciona, con él se pueden lograr descripciones detalladas de los estilos de respuesta, afectividad, operaciones cognitivas, motivación, percepción y relaciones interpersonales, con lo que se convierte en un poderoso instrumento para evaluar la dinámica de la personalidad (Weiner, 1992). 
Elección vocacional y personalidad en universitarios a través del psicodiagnóstico...

Para la codificación e interpretación se usó el Sistema Comprehensivo de Exner (1994), debido a que, con él, la prueba cumple con la mayor parte de las condiciones psicométricas que requiere una prueba psicológica, habiéndose demostrado también su utilidad para la comparación de grupos, y de poblaciones normales.

\section{Procedimiento}

Luego de administradas las pruebas de manera individual, a aquellos alumnos seleccionados que aceptaron participar voluntariamente en la investigación, se procedió a la codificación de los protocolos. Se seleccionaron 20 casos al azar que fueron codificados de manera independiente por tres jueces. Se obtuvo con ello un coeficiente de confiabilidad de .89. Una vez aceptada la codificación se obtuvo el Sumario Estructural de cada caso y se realizaron los análisis estadísticos pertinentes, siguiendo las agrupaciones del Sumario Estructural.

\section{Resultados}

En primer lugar se aplicó la prueba de bondad de ajuste de Kolmogorov Smirnov, con el fin de determinar el tipo de distribución de cada variable de la prueba y aplicar de acuerdo con ello los estadísticos pertinentes para la comparación de grupos. Se utilizaron el análisis de varianza paramétrico (ANOVA) y el análisis de varianza no paramétrico de Kruskal Wallis, de acuerdo con el tipo de variable. En ambos casos se trabajó con un alfa de .05 , considerándose además algunas tendencias importantes. También se realizó, para algunas variables un análisis de tabulación cruzada y la prueba de Chi Cuadrado.

En primer lugar, cabe resaltar que en los resultados obtenidos para el grupo total, encontramos marcadas diferencias con los datos normativos de Exner y en cambio mucha similitud con las normas chilenas (Vinet, Saiz y San Martín, 1995) y especialmente con las venezolanas (Ephraim, 1996). Esto conduce a tomar en consideración la variable cultural en la interpretación de los resultados. 
Antes de analizar las diferencias encontradas entre los cuatro grupos evaluados, señalaremos algunas de las características comunes. Se puede observar cierta tendencia en el grupo total a simplificar la percepción de los estímulos (Lambda $=1.12)$, asociada a cierto control intelectual y a evitar el procesamiento de emociones (Bajo porcentaje de Respuestas Complejas). Asimismo encontramos que el grupo dedica una gran cantidad de energía al procesamiento de la información ( $W=49 \%$ ), y una tendencia a presentar una elevada motivación de logro. Si bien se encuentra una adecuada adaptación al medio, destaca cierta tendencia a alejarse de lo convencional (calidad formal).

En la comparación entre grupos encontramos diferencias estadísticamente significativas $(p<.05)$ en las agrupaciones de Mediación cognitiva, Ideación, y Autopercepción, que se analizarán a continuación.

En la agrupación de Mediación cognitiva, relacionada a los procedimientos de traducción e identificación de la información recibida, la diferencia se encuentra en las respuestas $F+\%$, vinculadas al ajuste convencional. Mediante la prueba de ANOVA se obtuvo un $\mathrm{F}(3,116)$ $=3.92, p=.01$. Aquí, el grupo de ingeniería es el que presenta un puntaje significativamente más bajo que el de los otros grupos. En el Figura 1 se muestra la distribución de casos de acuerdo con los niveles esperados de $\mathrm{F}+\%$. Se está considerando un nivel alto si sobrepasa la media más una desviación estándar, que se asocia a hiperconvencionalidad; y un nivel bajo, si es inferior a la media menos una desviación estándar, que se relaciona con cierto alejamiento de lo convencional. 
Elección vocacional y personalidad en universitarios a través del psicodiagnóstico...

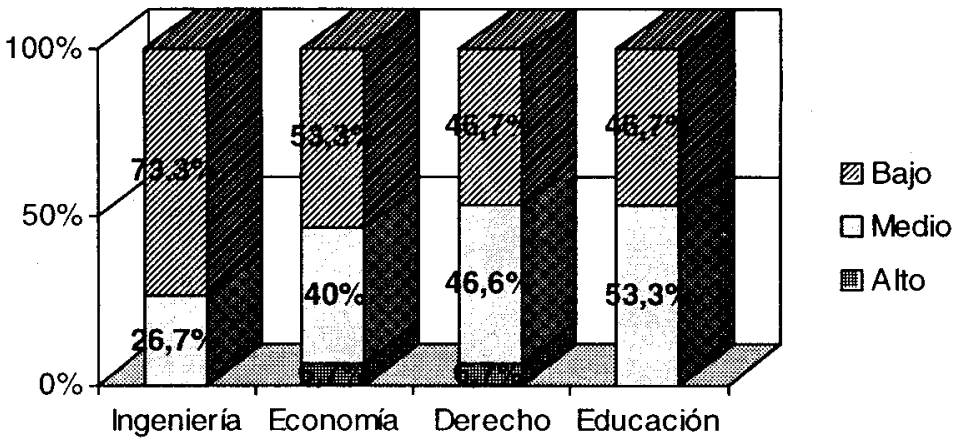

Figura 1. F+\% por Facultad

En la agrupación de Ideación, relacionada a la elaboración y construcción de nuevos conceptos, las diferencias se encontraron en la suma ponderada de los códigos especiales críticos (SumPond6); mediante la prueba de Chi cuadrado se obtuvo $X^{2}(6, \mathrm{~N}=120)=8.71, p<.05$. Los códigos especiales críticos se consideran un indicador de fallas lógicas en el curso de la ideación, la suma ponderada de estas variables representa un indicador global de dichas fallas por lo que en general se esperan niveles bajos. En el Figura 2 se presentan estos resultados considerándose un nivel alto si sobrepasa la media más una desviación estándar. Se observa que un $25 \%$ del grupo total presenta niveles altos, destacando el grupo de ingeniería, que estaría expresando así mayores dificultades en el curso de la ideación. 


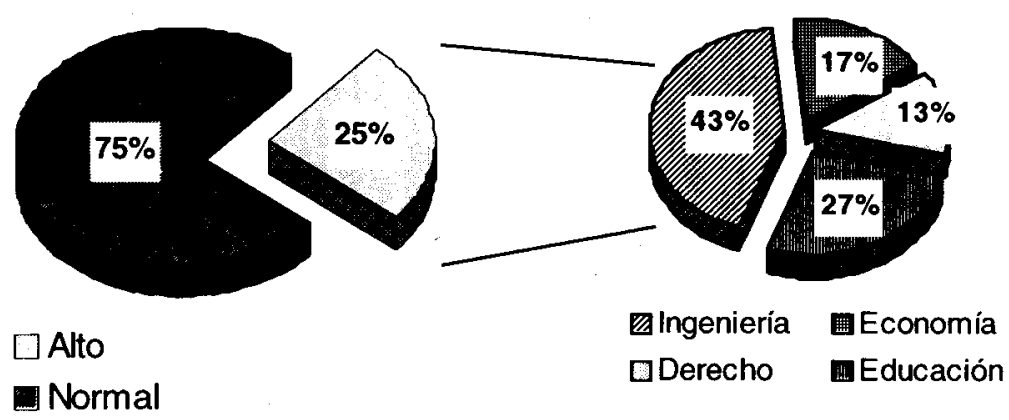

Figura 2. Suma Ponderada de Códigos Especiales Críticos.

Cabe mencionar que se realizó un análisis más fino sobre el tipo de Códigos especiales, agrupándolos de acuerdo con el tipo de trastorno que representan (Exner y Sendín, 1995). Se encontró que la mayor parte de códigos especiales correspondían a un trastorno leve o moderado, que en este caso representaría esencialmente un estilo particular, más que un trastorno específico.

En la agrupación de Autopercepción, relacionada al conjunto de conceptos $y$ actitudes que una persona ha construido sobre sí misma, se encontraron diferencias estadísticamente significativas en las respuestas de Forma Dimensión (FD) y en las de Vista (V), como puede observarse en el Cuadro 1. La FD representa el uso de la introspección y la capacidad para tomar distancia de los estímulos externos, y dada la etapa evolutiva de la muestra, podría relacionarse con el proceso de cambio o crecimiento personal. Su ausencia, no tiene mayor significado interpretativo. Las respuestas de Vista, adquieren importancia porque expresan componentes de desvalorización asociados con los procesos de introspección, es decir, una autocrítica negativa que puede producir sufrimiento psíquico. Las diferencias encontradas en ambos variables, señalan al grupo de Ingeniería con mayor cantidad de respuestas de este tipo. 
Elección vocacional y personalidad en universitarios a través del psicodiagnóstico...

Cuadro 1

Análisis de Varianza no Paramétrico (Kruskal Wallis) de Autopercepción (FD $y$ V)

\begin{tabular}{|c|c|c|c|c|c|c|}
\hline$\sqrt{2} \ln (19)$ & $(n-30)$ & $(\mathrm{n}-30)$ & $\begin{array}{l}\text { Deredio } \\
(n=30)\end{array}$ & $\begin{array}{r}\text { Dilicain } \\
(\mathrm{n}=30)\end{array}$ & 8 & $\sqrt{7}$ \\
\hline \multicolumn{7}{|l|}{ FD } \\
\hline Media & 0.73 & 0.57 & 0.17 & 0.37 & \multirow[t]{2}{*}{9.68} & \multirow[t]{2}{*}{.02} \\
\hline D.S. & 0.91 & 0.73 & 0.38 & 0.56 & & \\
\hline \multicolumn{7}{|l|}{$\mathrm{V}$} \\
\hline Media & 0.6 & 0.3 & 0.17 & 0.3 & \multirow[t]{2}{*}{8.3} & \multirow[t]{2}{*}{.04} \\
\hline D.S. & 0.77 & 0.53 & 0.38 & 0.7 & & \\
\hline
\end{tabular}

Nota. ' 3 grados de libertad en ambas variables.

Asimismo, encontramos toda una serie de diferencias que si bien no alcanzan el nivel estadístico propuesto de .05 , evidencian tendencias importantes.

Así en el grupo de Ingeniería se encuentra una mayor tendencia a la introversión, un $46.7 \%$ de casos presenta un estilo introversivo, y sólo un $6.7 \%$ de casos con un estilo extratensivo. Es asimismo el grupo que presenta una mayor cantidad de casos con un estilo excesivamente recopilador que se deja invadir fácilmente por la estimulación emocional $(26.7 \%$ de casos con un Lambda bajo, inferior a 0.5$)$.

El grupo de Derecho es el que presenta el promedio de Lambda más alto $(M=1.37)$, asociado a un estilo sobresimplificador con el que se evita procesar emociones, perdiéndose con ello aspectos esenciales del estímulo.

En la agrupación de Procesamiento de la Información, se encuentran tendencias importantes en este grupo, en la Actividad Organizativa (Zd) y en la Elaboración Cognitiva (DQ+) (Cuadro 2). 
Cuadro 2

Análisis de Varianza no paramétrico (Kruskal Wallis) de Procesamiento de la información ( $Z d$ y $D Q+$ )

\begin{tabular}{|c|c|c|c|c|c|c|}
\hline Variable & $\begin{array}{c}\text { lingenienta } \\
(n-30)\end{array}$ & $\begin{array}{l}\text { Economina } \\
(0-30)\end{array}$ & $\begin{array}{l}\text { Eercho } \\
(n=30)\end{array}$ & $\begin{array}{l}\text { Fducicion } \\
(n=30)\end{array}$ & $x^{2}$ & 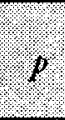 \\
\hline \multicolumn{7}{|l|}{$\mathrm{Zd}$} \\
\hline Media & -2.17 & -1.07 & -3.42 & -1.67 & 3.54 & .32 \\
\hline D.S. & 4.47 & 4.05 & 4.99 & 3.43 & & \\
\hline \multicolumn{7}{|l|}{$\mathrm{DQ}_{+}$} \\
\hline Media & 5.83 & 4.97 & 3.93 & 5.43 & 6.3 & .1 \\
\hline D.S. & 3.49 & 2.2 & 2.57 & 3.76 & & \\
\hline
\end{tabular}

Nota. ' 3 grados de libertad para ambas variables

La $\mathrm{Zd}$ representa un índice de la eficiencia con que se lleva a cabo el procesamiento de la información. En general un puntaje inferior a menos tres corresponde a un estilo hipoincorporador, que implica un examen poco cuidadoso del estímulo que lleva a perder eficacia. La DQ+, asociada a un modo más sofisticado de elaboración cognitiva del área de localización que se ha elegido, se presenta en el grupo de Derecho, en un nivel más bajo que en los otros grupos.

Asimismo, en la proporción W:M, es el grupo que presenta mayor cantidad de casos con un nivel de aspiraciones superior a los recursos actuales $(63.3 \%)$.

En la agrupación de Rasgos Afectivos, vinculados al manejo de las emociones, se encuentra en Derecho un alto porcentaje de casos de constricción afectiva, es decir con dificultades en la expresión o externalización de los afectos, expresados en la proporción Suma C': Suma Ponderada de Color, que se observa en el Cuadro 3. Lo esperado en esta proporción es que SumC' sea inferior a SumPondC. 
Elección vocacional y personalidad en universitarios a través del psicodiagnóstico...

Cuadro 3

Rasgos Afectivos (SumC': SumPondC)

\begin{tabular}{|c|c|c|c|c|}
\hline $\begin{array}{l}\text { SumCi } \\
\text { Sumponc }\end{array}$ & Ingerieria & Economita & Derecho & Rrinewion \\
\hline $\mathrm{C}^{\prime}<\mathrm{SPC}$ & $\begin{array}{c}80 \% \\
(n=24)\end{array}$ & $\begin{array}{l}76.7 \% \\
(\mathrm{n}=23)\end{array}$ & $\begin{array}{l}53.3 \% \\
(\mathrm{n}=16)\end{array}$ & $\begin{array}{l}76.7 \% \\
(\mathrm{n}=23)\end{array}$ \\
\hline $\mathrm{C}^{\prime}<\mathrm{SPC}$ & $\begin{array}{l}20 \% \\
(n=6)\end{array}$ & $\begin{array}{c}23.3 \% \\
(\mathrm{n}=7)\end{array}$ & $\begin{array}{l}46.7 \% \\
(n=14)\end{array}$ & $\begin{array}{c}23.3 \% \\
(n=7)\end{array}$ \\
\hline
\end{tabular}

Asimismo es el grupo que presenta un mayor porcentaje de casos con un nivel de respuestas complejas (Compl) por debajo de lo esperado, es decir menos del $20 \%$. Aquí se encuentra el $90 \%$ del grupo, que estaría presentando un estilo simplificador con dificultades para el manejo de estímulos emocionales complejos.

En la agrupación de Relaciones Interpersonales, el grupo de Derecho destaca por una menor cantidad de respuestas de Textura $(53.3 \%$ de casos sin respuesta de Textura), que se relacionan con las necesidades de contacto afectivo, su ausencia se asocia a dificultades en el establecimiento de contactos afectivos cercanos.

Finalmente es el grupo que presenta mayor cantidad de casos con un Indice de Inhabilidad Social (CDI) positivo (50\% de casos). Esto se relaciona con dificultades para enfrentarse a las demandas comunes del medio, se asocia a personas distantes y menos sensibles a las necesidades del otro, por lo tanto con mayores dificultades para todo lo relacional.

El grupo de Economia, destaca básicamente en las variables de localización, por su estilo de procesar la información (Figura 3). 


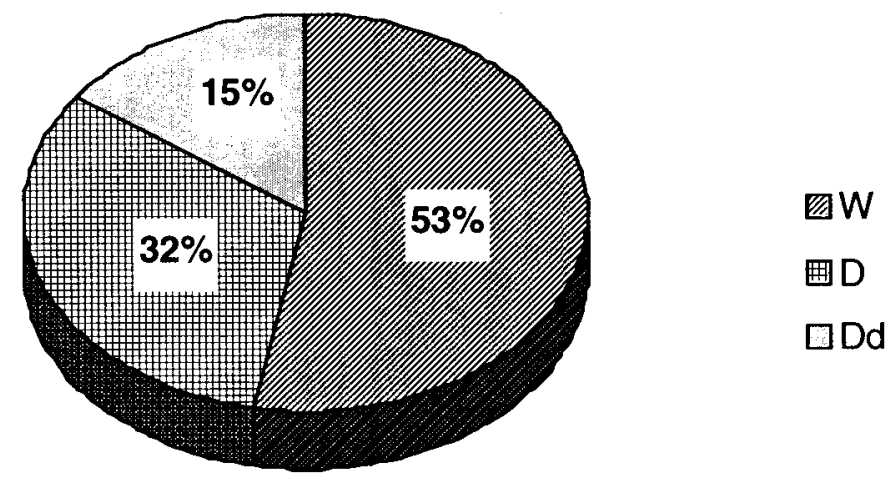

Figura 3. Variables de Localización en Economía

Se observa aquí, una tendencia a un enfoque totalizador de la realidad, con un marcado predominio de respuestas globales (W\%), asociadas a la capacidad para organizar los componentes del entorno abarcando todos sus elementos. Es asimismo el grupo que destaca por presentar mayor cantidad de casos con un tipo vivencial extratensivo $(30 \%)$.

El grupo de Educación, es el grupo que en general se presenta como más convencional. Alcanza un Lambda de 0.95 , dentro de lo esperado, como en la mayor parte de variables. La diferencia más saltante en este grupo, en relación con los otros, se encuentra en el nivel de motivación de logro, que se puede observar en la Figura 4. Se puede hablar de una motivación elevada, cuando la $\mathrm{Zf}$ es superior a la media, la W superior a $\mathrm{D}+\mathrm{Dd}$ y la W sube en la proporción W:M (Exner y Sendín, 1995). Lo contrario expresa una escasa motivación que se presenta en un $36.7 \%$ de casos de Educación. 
Elección vocacional y personalidad en universitarios a través del psicodiagnóstico...

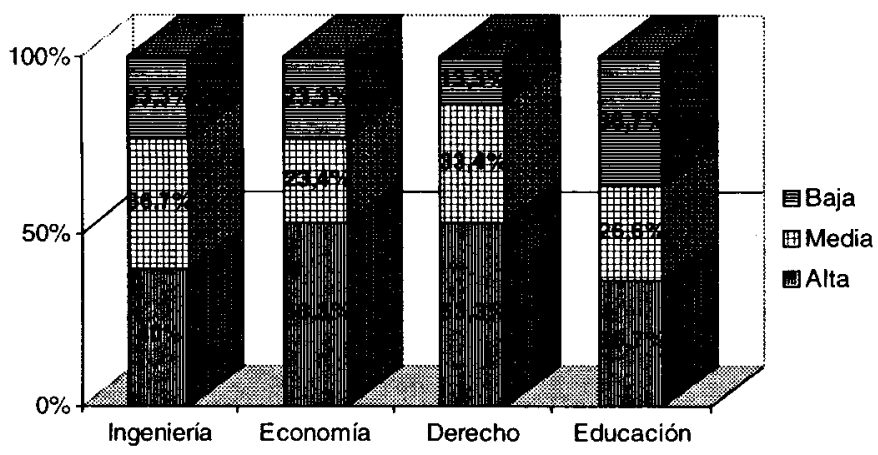

Gráfico 4. Nivel de Motivación por Facultad

\section{Discusión}

Un primer tema de discusión, surge a partir de las diferencias encontradas entre las normas de Exner y los puntajes totales del grupo, debido a que gran parte de la interpretación de los protocolos se basa en la comparación con los datos normativos, que son los que en última instancia definen lo esperado o adecuado y lo que se aleja de los patrones de normalidad en base a criterios estadísticos.

En Latinoamérica y en el mundo en general, se están realizando continuas investigaciones con el Psicodiagnóstico de Rorschach, con el fin de elaborar normas propias y de detectar diferencias que podrían deberse, no a desviaciones psicopatológicas, sino a aspectos culturales. Las semejanzas encontradas entre la muestra de este estudio y las normas venezolanas y chilenas, nos sugieren la existencia de características comunes a un grupo latinoamericano, que tendrían que ser tomadas en consideración.

Siguiendo a Ephraim (1996), pensamos que justamente éste es uno de los principales aportes del Psicodiagnóstico de Rorschach, ya que 
la riqueza de información que proporciona revela estilos de personalidad que permiten establecer comparaciones entre grupos. Lo mismo indican los hallazgos de Ráez (1998), quién afirma que el nivel educacional y el estatus socioeconómico son variables que siempre deben ser tomadas en cuenta en la investigación con Rorschach.

Las semejanzas encontradas en los cuatro grupos del presente estudio, revelan características comunes, asociadas probablemente a que se trata de un grupo muy homogéneo, todos los evaluados corresponden a una misma etapa de desarrollo, han pasado por un proceso de selección para ingresar a la universidad, han cumplido con las exigencias de la misma y se encuentran finalizando sus estudios. Podríamos suponer a partir de esto que el hecho de pertenecer a un mismo centro de estudios, es un elemento de importancia en la constitución de la identidad, más allá de la carrera elegida. Todos pertenecen a la Universidad " $X$ " que se constituye en su grupo de referencia y por lo tanto en un modo de definirse.

De acuerdo con Bohoslavsky (1984), así como no es casual la elección de una carrera, tampoco es casual la elección de la universidad en la que se desea estudiar, que además ejerce una influencia directa en los jóvenes. En este sentido Osipow (1991), considera, además que la exposición a las actividades y al clima mismo de la institución ejerce influencia en el comportamiento y en la personalidad del individuo.

Las diferencias estadísticas encontradas señalan principalmente al grupo de Ingeniería como el que se diferencia más de los otros tres. Se observa en ellos una mayor tendencia a alejarse de lo convencional y mayores dificultades en el curso de la ideación, muy relacionada con ese alejamiento de lo convencional. Del mismo modo es el grupo que muestra mayor tendencia a la desvalorización de sí mismos, asociada a una mayor introspección, que en este caso cobra un matiz de autocrítica negativa. Resulta significativo que se trate de la única facultad de Ciencias, ya que las otras tres si bien son facultades distintas, todas pertenecen al área de Letras. 
Elección vocacional y personalidad en universitarios a través del psicodiagnóstico...

Esto podría estar relacionado a lo descrito por Roe (1972), quién afirma que una dimensión esencial de la personalidad en la elección de carrera es la de trabajar con personas o evitar hacerlo. Las carreras de Letras que hemos investigado implican un mayor contacto con personas a diferencia de la de Ciencias que implica una mayor instrumentalización de los objetos y materias.

Es posible mencionar también el otro grupo de diferencias, que si bien no alcanza el nivel de significación estadística propuesto, denota claras tendencias en algunas características que además coinciden entre si, y que pueden permitirnos entender un poco más las diferencias anteriormente mencionadas.

El grupo de Ingeniería presenta una mayor tendencia a la introversión que puede estar relacionada al mayor uso de la introspección. Es asimismo el grupo que presenta una mayor cantidad de casos con un estilo excesivamente recopilador que se deja invadir fácilmente por la estimulación emocional; esto podría explicar, al menos en parte, las dificultades en torno a la ideación y el alejamiento de lo convencional, ya que existiría una mayor tendencia a captarlo todo, con la posibilidad de llegar a sentirse inundados por ello.

En un sentido distinto, el grupo de Derecho presenta una mayor constricción afectiva que dificulta el procesamiento de las emociones. Vinculado a esto, muestra una mayor necesidad de simplificar sus percepciones y de evitar dejarse invadir por sus emociones, probablemente asociado a la menor elaboración e integración de los estímulos. Es el grupo que presenta mayores dificultades en el control y modulación de los afectos y mayor dificultad en el establecimiento de contactos interpersonales más profundos.

Finalmente, el grupo de Economía muestra mayor tendencia a un enfoque totalizador en su percepción de los estímulos, con el consiguiente descuido de los aspectos prácricos y concretos de la experiencia. Es asimismo, el grupo que presenta mayor tendencia a un estilo vivencial extratensivo. El grupo de Educación es el que en general se ubica de 
un modo más convencional, más apegado a la norma. Es asimismo el grupo más homogéneo, destacando únicamente por su escasa motivación de logro en proporción a los otros grupos.

A partir de todo lo descrito, podemos afirmar que si bien existen muchos elementos en común en las cuatro facultades, es evidente que las diferencias existen, ellas señalan básicamente tendencias o estilos de respuesta distintos en cada una de las facultades. Uno de los hallazgos más saltantes lo constituye el grupo de Ingeniería, que nos permite suponer que si existen diferencias saltantes entre las personas que optan por carreras de Ciencias o de Letras, que seria el tipo de actividad de marca la principal diferencia. Todo esto nos permite confirmar que sí existe una relación entre Elección Vocacional y Personalidad, ya que existen características especificas que predominan en cada una de las carreras que hemos investigado, a pesar de no alcanzar muchas de ellas el nivel de significación estadística propuesto.

Es importante tomar en consideración que además de la carrera elegida, el tipo de labor que se pretende desempeñar implica también toda una serie de matices distintos, por lo que las diferencias halladas representan únicamente tendencias generales. Así lo demuestran también los estudios de Weinrach (1996) y Zachar y Leong (1992).

Por otro lado, la presente investigación nos permite confirmar la utilidad del Psicodiagnóstico de Rorschach, para la comparación de grupos, ya que facilita un acercamiento a aquellos matices de la personalidad que escapan a otro tipo de instrumentos que evalúan rasgos más específicos. El valor de la técnica, radica justamente, en poder aproximarnos a la "estructura y dinámica de la personalidad" en conjunto y poder describir a partir de ello estilos o tendencias. 
Elección vocacional y personalidad en universitarios a través del psicodiagnóstico...

\section{Referencias}

Bohoslavsky, R. (1984). Orientación vocacional: una estrategia clínica. Buenos Aires: Nueva Visión.

Casullo, M., Cayssials, A., Liporace, M., De Diuk, L., Arce, J. y Alvarez, L. (1996). Proyecto de vida y decisión vocacional. Buenos Aires: Paidós.

Ephraim, D. (1996). El método de Rorschach en la actualidad. Caracas: Monteávila.

Erikson, E. (1993). El ciclo vital completado. México: Paidós.

Exner, J. (1994). El Rorschach. Un Sistema Comprehensivo. (Vol. 1) Madrid: Psimática.

Exner, J. (1995). Manual de Codificación del Rorschach para el Sistema Comprehensivo. Madrid: Psimática.

Exner, J. y Sendín, C. (1995). Manual de Interpretación del Rorschach para el Sistema Comprehensivo. Madrid: Psimática.

Furnham, A. y Stringfield, P. (1993). Personality and occupational behavior: Myers-Briggs Type Indicator Correlates of managerial practices in two cultures. Human Relations, 46, 7, 827-848.

Gottfredson, G., Jones, E. y Holland, J. (1993). Personality and Vocational Interests: The Relation of Holland's Six Interest Dimensions to Five Robust Dimensions of Personality. Journal of Counseling Psychology, 40, 4, 518-524.

Hernández, R., Fernández, C. y Baptista, P. (1991). Metodología de La Investigación. México: Mc Graw Hill.

Holland, J. (1973). Making vocational choices: a theory of careers. Nueva Jersey: Prentice Hall.

Holland, J. (1996). Exploring Careers with a Typology. American Psychologist, 51, 397-406.

Khan, S. y Alvi, S. (1991). The Structure of Holland's typology. A study in a Non-Western Culture. Journal of Cross cultural Psychology, 22, 2, 283-292. 
Nordvik, H. (1996). Relationships between Holland's vocational typology, Schein's career anchors and Myers-Briggs' types. Journal of Occupational and Organizational Psychology, 69, 263-275.

Osipow, S. (1991). Teorias sobre la elección de carreras. México: Trillas. Ráez, M. (1998). Personality development of women leaders: assessment studies in peruvian urban and poverty areas. Nimega: KUN.

Ráez, M., Rossel, Z., Niño de Guzmán, 1, Martínez, P. y Figueroa, M. (1986). El Psicodiagnóstico de Rorschach como instrumento evaluador de grupos. Revista de Psicologia, 4, 1, 83-110.

Roe, A. (1972). Psicología de las profesiones. Madrid: Marova.

Super, D. (1977). Psicología de los intereses y las vocaciones. Buenos Aires: Kapelusz.

Tokar, D. y Swanson, J. (1995). Evaluation of the correspondence between Holland's Vocational Personality Typology and the Five Factor Model of Personality. Journal of vocational behavior, 46, 89-108.

Vinet, E., Saiz, J. y San Martín, C. (1995). Necesidad de normas nacionales en el Sistema Comprehensivo del Psicodiagnóstico de Roschach: el caso de Chile. Revista Iberoamericana de Diagnóstico y Evaluación Psicológica, 2, 189-201.

Weiner, 1. (1992). Métodos en Psicologia Clínica. México: Limusa.

Weinrach, S. (1996). The Psychological and Vocational Interest Patterns of Donald Super and John Holland. Journal of Counseling \& Development, 75, 5-16.

Zachar, P. y Leong, F. (1992). A problem of Personality: Scientist and Practitioner differences in Psychology. Journal of Personality, 60, 3, 665-677. 